\title{
Educação em saúde para cuidado humanizado ao idoso com Alzheimer: extensão em tempo de pandemia
}

\author{
Nathan Marcondes Freitas Leite \\ nathanmarcondes@gmail.com \\ Jeiel Rocha Oliveira da Silva \\ jeielrochao@gmail.com \\ Patrícia Cristiane Gibbert \\ patriciagibbert123@gmail.com \\ Stella Rodrigues Barros do Nascimento \\ stella_rbn@hotmail.com \\ Neudson Johnson Martinho \\ neudsonjm@hotmail.com
}

\section{Resumo}

A Doença de Alzheimer (DA) é a patologia neurodegenerativa mais frequente associada à idade. Suas manifestações cognitivas e neuropsiquiátricas resultam na deficiência progressiva e eventual incapacitação, causando um sofrimento expressivo ao seu portador e a todos envolvidos, uma vez que pode levar à sua sobrecarga física e emocional. 0 objetivo deste trabalho é demonstrar a possibilidade de educação em saúde online com impactos positivos no que tange a novas aprendizagens quanto à DA. Foi elaborado um vídeo sobre a doença e o cuidado humanizado ao portador, sendo avaliado o impacto deste. Verificou-se que, a despeito do isolamento social, houve impacto positivo para os participantes avaliados.

\section{Palavras-chave}

Educação em Saúde; Doença de Alzheimer; Saúde do Idoso. 


\section{Introdução}

A Doença de Alzheimer (DA) é uma neuropatologia crônica e progressiva, caracterizada por alterações nas funções cognitivas, como a memória, capacidade afetiva e comportamento, perda da autonomia e da capacidade de participar de interações (BARBE et al., 2018). Além das dificuldades de atenção e fluência verbal, outras funções cognitivas deterioram à medida que a patologia evolui. Esses sintomas são frequentemente acompanhados por distúrbios comportamentais, em que o paciente torna-se mais agressivo, com possíveis episódios de alucinação, hiperatividade, irritabilidade e, até mesmo, quadros depressivos (SERENIKI e VITAL, 2008).

A DA apresenta três estágios, em que o inicial é marcado por esquecimentos e perdas sutis da memória e dificuldades no trabalho, ocasionando frequente confusão com estados depressivos. 0 estágio intermediário, marcado pela progressão da doença, caracteriza-se pela perda mais acentuada da memória e incapacidade de realizar tarefas complexas, como cálculos e planejamentos. Em estágios mais avançados, em razão do comprometimento da sua capacidade funcional, o portador de Alzheimer tem dificuldades para se alimentar, vestir-se e banhar-se, sendo caracterizados pela restrição ao leito (DE ABREU; FORLENZA; DE BARROS, 2005; RUIZFERNÁNDEZ, 2019).

A demência, causada principalmente por DA, é muito comum em idosos octagenários, parte da população brasileira que mais cresce no Brasil (CHAIMOWICZ, 2013). Em contrapartida, ainda prevalece no país o suporte informal ao idoso com DA, o que sobrecarrega a família ou outra pessoa despreparada, em razão da ausência de uma rede de suporte adequada (LOUREIRO, 2014).

Devido à progressiva diminuição das capacidades cognitivas, desde a fase inicial da doença é necessário um acompanhamento constante da pessoa com DA nos seus afazeres diários. Assim, na maioria das vezes, um membro da família ou amigo se torna responsável pelo manejo dessa atenção, o qual é obrigado a zelar pela saúde física, psicológica e social do seu parente adoecido. A dinâmica do cuidado varia conforme cada grupo familiar, adquirindo diferentes formas de atenção com base não somente na personalidade do familiar cuidador e na concepção que este tenha sobre o cuidado, mas também em razão das oportunidades referentes às redes de apoio e estratégias de enfrentamento ofertadas. Dessa forma, por muitas vezes não receber nenhum preparo ou treinamento específico, o atendimento às necessidades do ente cuidado é comprometido (VIZZACHI, 2015). 
Além disso, o cuidador precisa adaptar a sua rotina ao portador de Alzheimer, oferecendo atendimento constante para atividades relacionadas à alimentação, higiene e conforto. Contudo, devido à teimosia da pessoa cuidada, à progressão da doença e às dificuldades desse encargo, o cuidador pode apresentar variações de humor e exacerbação de sentimentos de raiva e tristeza. Somado a isso, frequentemente há a necessidade da abdicação das suas atividades particulares, sociais e econômicas, provocando um desgaste emocional e afetando a sua saúde psíquica (KARLAWISH, 2014; KUCMANSKI, 2016).

Portanto, a adição desses problemas sociais, econômicos, físicos e psicológicos caracteriza o quadro de sobrecarga do cuidador. Entretanto, essa sobrecarga varia conforme a idade, escolaridade e sexo do cuidador, dependendo também do nível de progressão da enfermidade, do tempo empregado nesse encargo, dos sintomas manifestados e do ponto de dependência da pessoa cuidada. Ademais, como essas pessoas presenciam o portador de DA "morrer duas vezes", sendo a primeira morte a da mente, eles tomam decisões eticamente carregadas em razão da vivência dessa morbidade junto a quem recebe o cuidado (KARLAWISH, 2014; RUIZ-FERNÁNDEZ, 2019).

Sob essa perspectiva, essa sobrecarga significativa e as alterações psicopatológicas decorrentes tornam o cuidador menos disponível para prestar o seu papel, não permitindo, portanto, o adequado atendimento às necessidades de ambos. Um cuidador exausto não é capaz de acompanhar o paciente, o que implica uma série de agravos para atividades cotidianas, interferindo na qualidade de vida do portador de DA em razão da aceleração da deterioração física e mental deste (BARBE et.al, 2018; GARZÓN-MALDONADO, 2017).

Dessa forma, o portador de DA e os cuidadores precisam de assistência à saúde para que cada um conviva com dignidade e bem-estar (KARLAWISH, 2014). A humanização do cuidado precede pela ciência de que o cuidador e o portador necessitam de atenção integral de uma equipe multiprofissional e interprofissional, a qual inclua médicos generalistas, clínicos e de família que saibam utilizar das habilidades de comunicação, empatia, responsabilidade para com o portador de DA e seus familiares e do entendimento das reais necessidades dessas pessoas (MANSO, 2019). É preciso, também, que o cuidador saiba como realizar o cuidado conforme os declínios de saúde que a pessoa com DA vivenciará para que não seja surpreendido e consiga atender as necessidades de seu ente querido, mas para que também consiga disponibilizar um período para as suas atividades pessoais.

Ademais, humanizar o cuidado também inclui a consideração pela subjetividade dos indivíduos envolvidos, o que se relaciona a um agir de uma maneira mais humanizada e empática com o idoso, tratando-o pelo nome, não o infantilizando, agindo para diminuir seu 
sofrimento e melhorar sua qualidade de vida, sendo criativo para atender o paciente e lidar com seus devaneios. Assim, o cuidado deve ser completo, considerando-se o atendimento às necessidades básicas do paciente de forma que este seja reconstruído como ser humano, devendo, então, despertar os autoconceitos do portador de DA e reconstruir sua identidade pessoal (LI, 2002).

Nesse sentido, uma das alternativas mais importantes para assegurar certa autonomia a ambos, é a ação educativa. A educação em saúde ocorre de forma dinâmica e tem o objetivo de capacitar o indivíduo, a fim de promover qualidade de vida e dignidade. Ressalta-se que os participantes de educação em saúde podem aceitar ou negar as mudanças propostas e, assim, as ações devem estimular a indagação e reflexão sobre a temática (MARTINS, 2007).

Dessa forma, foi realizada uma ação educativa, por meio de curta-metragem intitulada "A Doença de Alzheimer e a humanização do cuidado" e, em seguida, o presente estudo, a fim de entender como a sociedade, possíveis cuidadores ou conhecedores de pessoas portadoras de DA, e graduandos da saúde, entendem os vieses social e evolutivo da patologia, e, ainda, para sensibilizar esses participantes frente às dificuldades enfrentadas por cuidadores e portador da doença.

Portanto, o objetivo desse trabalho é promover educação em saúde sobre os cuidados ao paciente com DA e ao seu cuidador, de modo a proporcionar uma melhor qualidade de vida, respaldada nos valores e prerrogativas da humanização, ajudando-os a ultrapassar o limite dessa fase da vida com dignidade. Assim, segundo CELICH E BATISTELLA (2007), os idosos e seus familiares podem compartilhar de suas emoções e angústias.

\section{Metodologia}

Trata-se de um estudo do tipo descritivo, com abordagem quantitativa. Segundo Tobar e Yalour (2001), estudos descritivos são aqueles em que se observa os fenômenos, registra-os, analisa-os e, então, os classifica e interpreta, sem que haja manipulação pelo pesquisador. Ou seja, eles apenas são descritos como se apresentam.

Diehl (2004) afirma que a pesquisa quantitativa se caracteriza pelo uso da quantificação, como o próprio nome já refere. Sendo este processo tanto na coleta, quanto no tratamento das informações, utilizando-se técnicas estatísticas, visando obter resultados que evitem possíveis distorções de análise e interpretação, possibilitando uma possível margem de segurança. 
Richardson (1989) difere a abordagem qualitativa da quantitativa, em princípio porque a qualitativa não emprega um instrumento estatístico como base na análise de um problema, não pretendendo medir ou enumerar categorias. Trabalha com subjetividades e fenômenos sociais.

Esse estudo é um recorte do projeto aprovado pelo Comitê de Ética em Pesquisa (CEP) "Aprendendo e Ensinando a Conviver e Cuidar de Idosos com Doença de Alzheimer (DA)", desenvolvido pelo grupo Pesquisas Multiprofissionais em Educação e Tecnologias em Saúde (PEMEDUTS). Esse projeto de extensão visa possibilitar a educação em saúde como meio para a identificação precoce de sinais e sintomas sugestivos da DA, como também, favorecer a aprendizagem quanto aos cuidados necessários a serem dispensados aos portadores dessa doença.

Em consequência da Pandemia de COVID 19 as ações do projeto foram executadas via online durante os meses de julho e agosto de 2020, caracterizadas por reuniões de planejamento através do Google Meet e elaboração de um vídeo na plataforma de criação de vídeos animados "PowToon" a respeito da temática do projeto. 0 vídeo foi publicado na plataforma Youtube e compartilhado via redes sociais. Este foi subsidiado na literatura norteadora do projeto em epígrafe.

Para avaliar o impacto do vídeo foi elaborado um formulário na plataforma do Google Forms, composto por 13 perguntas, visando verificar os conhecimentos das pessoas acerca do tema abordado. Ele ficou disponível por um período de 24 horas e foi preenchido por um total de 28 pessoas. Os resultados foram analisados quantitativamente, conforme supracitado.

\section{Resultados e discussão}

Vinte e oito (28) participantes que assistiram o vídeo responderam o formulário no Google Forms, sendo que destes, 67,9\% se encontram na faixa etária entre 19 a 24 anos. Ao serem questionados se conviviam com alguém portador de DA, somente 10,7\% responderam que sim e os demais afirmaram apenas conhecer cuidadores de idosos portadores da mesma. Na amostra de respondentes não havia nenhum que tenha sido ou que seja cuidador.

50\% dos respondentes afirmaram que já conheciam o termo "Humanização do Cuidado", apesar de muitos nunca terem escutado uma explicação teórica a respeito do que significa a humanização, descreveram com considerável precisão este conceito, utilizando expressões como "carinho", "entendimento de forma integral do ser humano", "empatia", "tratamento humano" e "respeito". Já 89,3\% deram 5 numa escala de 1 a 5 para a questão sobre o quão preciso é uma equipe multiprofissional de saúde para uma pessoa portadora de DA, sendo que 5 representa 
"maior necessidade". Isso demonstra que boa parte das pessoas possui um entendimento adequado a respeito do que significa a humanização e consideram que a presença de uma equipe multiprofissional é necessária para atingir o objetivo de um cuidado mais completo. Também explicita que, apesar de ser ainda pouco aplicado no Brasil, esse tipo de cuidado pode ser facilmente implementado com apoio popular.

Apenas 7,1\% dos entrevistados afirmaram ter presenciado algum momento de violação dos direitos humanos de uma pessoa com Doença de Alzheimer. As situações foram descritas como "a pessoa teve sua opinião completamente desprezada" e "o familiar achou que o paciente estava fazendo de propósito". Em ambos os casos, é possível perceber que a falta de conhecimento a respeito da doença e do processo de adoecimento fazem com que o doente seja colocado em uma situação de vulnerabilidade psicológica e social, não sendo tratado como um indivíduo único pelo cuidador ou por familiares. Além disso, a sobrecarga física e emocional ocasionada por essa falta de preparo, apoio e treinamento específico pode levar a um mal-estar entre o cuidador e o doente, impactando diretamente a qualidade de vida de ambos (CELICH e BATISTELLA, 2007).

Além disso, 21\% dos participantes relataram ter um conhecimento teórico a respeito da fisiopatologia da doença, $3 \%$ afirmaram ter conhecimento amplo devido à sua formação anterior, assim como, 3\% referiram que o conhecimento prévio que tinha se dava pela sua convivência com uma portadora da DA.

As respostas depois de assistirem foram $100 \%$ positivas, pois todos relataram ter adquirido conhecimentos a partir do mesmo que possibilitou compreender melhor o desenvolvimento da doença e como ciclo social do portador é afetado. Isso demonstra que conseguimos atingir nosso objetivo de educação em saúde ainda que por meio de ferramentas virtuais. Além do mais, com um vídeo divulgado em uma grande plataforma como o YouTube, torna-se possível atingir um número muito maior de pessoas do que seria possível por capacitações presenciais.

A apresentação na forma de um curta-metragem animado também foi de crucial importância para que o conhecimento fosse transmitido de forma lúdica e, assim, pudemos instigar a reflexão a respeito de aspectos da DA que são pouco divulgados pela mídia em geral, como a questão da personalidade do indivíduo e os impactos para os cuidadores e familiares. Os respondentes apontaram grandes mudanças de paradigmas, dentre as quais destacam-se a aprendizagem quanto à manutenção de parte personalidade do doente, à possível reintegração social da pessoa afetada e quanto à importância do cuidado que deve ser prestado ao cuidador e família do portador, uma vez que também estes são afetados física e emocionalmente ao cuidar 
do idoso com DA. Assim, vê-se que cuidar é o ato de assistir alguém ou prestar serviços quando esse necessita, sendo uma atividade complexa, de características multidimensionais, das quais destacam-se as dimensões éticas, psicológicas, sociais, físicas, tendo seus aspectos clínicos, técnicos e comunitários (CELICH e BATISTELLA, 2007).

Outras aprendizagens apontadas foram relacionadas à forma de comunicação com os portadores, os estágios de evolução da doença e quanto às estatísticas relacionadas à mesma. Apenas dois do total de respondentes (7\%) relataram após assistirem o vídeo, que seus conhecimentos continuaram "rasos" ou "medianos". Esse fato coloca nosso vídeo em uma taxa de aproximadamente $93 \%$ de sucesso frente aos objetivos previamente determinados.

Um relato em especial merece destaque: um participante pontuou que "achava que para cuidar de alguém com DA, bastava dar os medicamentos". Esse relato reforça o impacto que a falta de conhecimento tem sobre a qualidade do cuidado prestado aos idosos portadores desta doença. No entanto, alguns dos entrevistados responderam que entendiam a humanização como importante, apesar de não compreenderem seu completo significado, enquanto outros, afirmaram saber da "importância de cuidar do psicológico tanto do portador quanto do cuidador".

A maioria dos respondentes do formulário, afirmaram que, a partir do mesmo, passaram a compreender a importância de se ter um olhar holístico e humanizado para com o portador, cuidador e família, uma vez todos ao conviverem com ele são afetados biopsicossocialmente pelas demandas de cuidado que este requer. Sendo necessário um olhar e ações dos profissionais de saúde que visem contribuir para qualidade de vida de todos os envolvidos, para que o portador também receba suporte e possa usufruir da melhor vivência possível enquanto presta seus cuidados ao doente.

\section{Considerações Finais}

A Doença de Alzheimer afeta amplos espectros da vida do seu portador e daqueles que convivem com ele. Tendo em vista que há uma inimaginável sobrecarga de trabalho que se torna progressivamente maior conforme o idoso perde sua autonomia e necessita de atenção constante. Além do mais, como no Brasil a condição econômica das famílias em geral não permite a contratação de um cuidador especializado e, como há escassez de casas de repouso especializadas para pessoas com demência, a sobrecarga econômica, emocional e social que aflige as famílias do portador da doença é ainda mais evidente. 
Tendo isso em vista, é preciso realizar atividades de educação em saúde que permitam o entendimento da doença como um todo, focalizando o indivíduo afetado, sua vivência e aqueles que são afetados indiretamente pela DA. Pois somente a partir dessas ações é possível levantar questionamentos acerca do que essas pessoas estão passando e incentivar um olhar mais humano sobre as mesmas, permitindo um verdadeiro incremento na qualidade de vida dos idosos e cuidadores envolvidos pela DA.

Ademais, consideramos que ações extensionistas que trabalham com educação em saúde sobre cuidados não farmacológicos com portadores de DA, seus familiares e cuidadores, devem ser estimuladas nos diversos cursos da área da saúde existentes no ambiente universitário. E, principalmente, que busquem trabalhar a interprofissionalidade, para que os futuros profissionais possam compreender a importância e a necessidade do trabalho interprofissional nesta dimensão do cuidar humano.

As respostas obtidas com o formulário disponibilizado no Google Forms vêm corroborar a necessidade de mudanças curriculares na formação médica e de outros cursos da área da saúde, no sentido de possibilitar aos alunos uma educação interprofissional e, consequentemente, uma atuação mais humanizada, onde o trabalho em equipe seja inserido no ensino teórico e prático. Assim, é possível que ocorram momentos de aprendizagem envolvendo diversos cursos da área da saúde em diálogos e práticas colaborativas, de modo específico no contexto do cuidado ao portador de DA.

No Brasil ainda é prevalente a formação uniprofissional e corporativa, dentro de "caixinhas", seccionada e positivista, a qual não contempla a formação acadêmica como uma relação entre os diversos cursos e profissionais de forma complementar, respeitosa e horizontal. A tendência mundial é a educação e o trabalho interprofissional. Este movimento está chegando ao Brasil e requer dos cursos um novo modus faciendi e modus operandi na formação, em especial, da medicina.

O currículo deve priorizar as DCN 2014, as quais são revolucionárias e priorizam uma formação mais humanizada e interprofissional, corroborando para a importância da tríade ensino-pesquisa e extensão durante todo o processo de formação profissional. Porém, essa DCN precisa ser materializada na sala de aula, nas relações docente-docente e docente-discente dentro e fora dos muros da faculdade e da universidade. Somente quando estas mudanças realmente ocorrerem, teremos trabalho em equipe de forma efetiva nos espaços de trabalho e um cuidado mais humanizado ao indivíduo, pessoa e comunidade. 


\section{Referências}

BARBE, Coralie et al. Factors associated with quality of life in patients with Alzheimer's disease. BMC Geriatrics, 2018, v. 18, n. 1, p. 1-9. Available from:

<https://bmcgeriatr.biomedcentral.com/articles/10.1186/s12877-018-0855-7>. Access on: 15 nov. 2020.

CELICH, Kátia e BATISTELLA, Maribel. Ser cuidador familiar do portador de doença de Alzheimer: vivências e sentimentos desvelados. Cogitare Enfermagem, v. 12, n. 2, p. 143-149, 2007. Disponível em: <http://dx.doi.org/10.5380/ce.v12i2.9821>. Acesso em: 15 nov. 2019.

CHAIMOWICZ, Flávio. Saúde do idoso. Belo Horizonte: NESCON UFMG, 2013. Disponível em: <https://www.nescon.medicina.ufmg.br/biblioteca/imagem/3836.pdf>. Acesso em: 19 nov. 2020.

DE ABREU, Izabella; FORLENZA, Orestes; DE BARROS, Hélio. Demência de Alzheimer: Correlação entre memória e autonomia. Revista de Psiquiatria Clínica, v. 32, n. 3, p. 131-136, 2005. Disponível em:<https://doi.org/10.1590/S0101-60832005000300005>. Acesso em: 10 nov. 2020.

GARZÓN-MALDONADO, Francisco, et al. Calidad de vida relacionada con la salud en cuidadores de pacientes con enfermedad de Alzheimer. Neurología, v. 32, n. 8, p. 508-515, 2017. Disponível em: <https://doi.org/10.1016/j.nrl.2016.02.023>. Acesso em: 10 nov. 2020.

LI, Ruobing; ORLEANS, Myron. Personhood in a World of Forgetfulness: An Ethnography of the Self-Process Among Alzheimer's Patients. Journal of Aging and Identity, v. 7, n. 4, p. 227-244, 2002. Disponível em: <https://doi.org/10.1023/A:1020709504186>. Acesso em: 11 nov. 2020.

KARLAWISH, Jason. How are we going to live with Alzheimer's disease? Health Affairs, v. 33, n. 4, p. 541-546, 2014. Available from: <https://doi.org/10.1377/hlthaff.2014.0089>. Access on: 14 nov. 2020.

KUCMANSKI, Luciane Salete et al. Alzheimer's disease: challenges faced by family caregivers. Rev. bras. geriatr. gerontol., v. 19, n. 6, p. 1022-1029, 2016. Available from: <http://dx.doi.org/10.1590/1981-22562016019.150162>. Access on: 15 nov. 2020.

LOUREIRO, Lara de Sá Neves et al. Sobrecarga em cuidadores familiares de idosos: associação com características do idoso e demanda de cuidado. Brasília: Rev. bras. enferm., v. 67, n. 2, p. 227- 232, 2014. Disponível em: <http://dx.doi.org/10.5935/0034-7167.20140030>. Acesso em: 10 nov. 2020.

MANSO, Maria Elisa Gonzalez. Cuidado centrado na pessoa para indivíduos com demência. Rev. Longeviver, v. 1, n. 1, p. 75-84, 2019. Disponível em:

$<$ https://revistalongeviver.com.br/index.php/revistaportal/article/viewFile/797/856>. Acesso em: 15 nov. 2020.

RUIZ-FERNÁNDEZ, María Dolores et al. Predictor Factors of Perceived Health in Family Caregivers of People Diagnosed with Mild or Moderate Alzheimer's Disease. Int. J. Environ. Res. Public Health, v. 16, n. 19, p. 3762, 2019. Available from:

<https://doi.org/10.3390/ijerph16193762>. Access on: 11 nov. 2020. 
SERENIKI, Adriana e VITAL, Maria Aparecida Barbato Frazão. A doença de Alzheimer: aspectos fisiopatológicos e farmacológicos. Rev. psiquiatr. Rio Gd. Sul [online], v. 30, n. 1, 2008. Disponível em: <https://doi.org/10.1590/S0101-81082008000200002>. Acesso em: 15 nov. 2020.

VIZZACHI, Barbara Alana et al. Dinâmica familiar frente ao Alzheimer em um de seus membros. Rev. esc. enferm. USP, v. 49, n. 6, p. 931-936, 2015. Disponível em:

<https://doi.org/10.1590/s0080-623420150000600008>. Acesso em: 15 nov. 2020.

BRASIL, Ministério da Saúde. 0 que é coronavírus? Brasília, 2020. Disponível em:

https://coronavirus.saude.gov.br/ 
Health education for humanized care for the elderly with Alzheimer: extension in times of pandemic

\begin{abstract}
Alzheimer's disease (AD) is the most frequent neurodegenerative pathology associated with age. Its cognitive and neuropsychiatric manifestations result in progressive disability and eventual incapacitation, causing significant suffering to the patient and everyone involved, as it leads to their physical and emotional overload. The objective of this work is to demonstrate the possibility of online health education with positive impacts in terms of new learning about $\mathrm{AD}$. A video about the disease and humanized care for the patient was made, and its impact was assessed. It was found that, despite social isolation, there was a positive impact for the evaluated participants.
\end{abstract}

\section{Keywords}

Health education; Alzheimer Disease; Health of the Elderly.
Educación en salud para el cuidado humanizado de los ancianos con Alzheimer: extensión en tiempo de pandemia

\section{Resumen}

La enfermedad de Alzheimer (EA) es la patología neurodegenerativa más frecuente asociada a la edad. Sus manifestaciones cognitivas $y$ neuropsiquiátricas resultan en una discapacidad progresiva y eventual discapacidad, provocando un sufrimiento significativo al paciente y a todos los involucrados, ya que conduce a su sobrecarga física y emocional. El objetivo de este trabajo es demostrar la posibilidad de una educación en salud online con impactos positivos en términos de nuevos aprendizajes sobre la EA. Se elaboró un video sobre la enfermedad y la atención humanizada al paciente y se evaluó su impacto. Se encontró que, a pesar del aislamiento social, hubo un impacto positivo para los participantes evaluados.

\section{Palabras clave}

Educación en Salud; Enfermedad de Alzheimer; Salud del Anciano. 